\title{
Optoelectronic evaluation of indirect dental veneers interfaces
}

\begin{abstract}
A.-C. Măroiu, M. Romînu, C. Sinescu, M.-L. Negruțiu, F. Topală, et al.
\end{abstract}

\begin{abstract}
A.-C. Măroiu, M. Romînu, C. Sinescu, M.-L. Negruțiu, F. Topală, V.-F. Duma, A. Bradu, A. Gh. Podoleanu, "Optoelectronic evaluation of indirect dental veneers interfaces," Proc. SPIE 10831, Seventh International Conference on Lasers in Medicine, 1083100 (10 August 2018); doi: $10.1117 / 12.2282648$
\end{abstract}

Event: Seventh International Conference on Lasers in Medicine, 2017, Timisoara, Romania 


\title{
Optoelectronic evaluation of indirect dental veneers interfaces
}

\author{
A.-C. Măroiu*a, M. Romînu ${ }^{a}$, C. Sinescu ${ }^{a}$, M.-L. Negruțiu ${ }^{a}$, F. Topalăa \\ V.-F. Duma ${ }^{\text {b,c }}$, A. Bradu ${ }^{d}$, A. Gh. Podoleanu ${ }^{\text {d }}$ \\ amaging Group, Faculty of Dental Medicine, "Victor Babes" University of Medicine and Pharmacy \\ of Timisoara, 2 Eftimie Murgu Square, 300041 Timișoara, Romania \\ b3OM Optomechatronics Group, “Aurel Vlaicu” University of Arad, 77 Revolutiei Ave., 310130 \\ Arad, Romania \\ ${ }^{\mathrm{c} D}$ Doctoral School, Polytechnics University of Timisoara, 1 Mihai Viteazu Ave., 300222 Timisoara, \\ Romania \\ dApplied Optics Group, University of Kent, Canterbury, CT2 7NZ, United Kingdom
}

\begin{abstract}
Dental indirect veneers have become the most functional and cost-effective method for providing high-aesthetic results whenever smile design enhancement is required. Yet, clinical failures have been reported due to the detachment of the veneers from the dental hard tissues, as well as to chipping and microleakage. Many experimental studies have been conducted in order to identify clinical and technical solutions for enhancing the adhesive and biomechanical properties of the veneers, by promoting the same classical, linear marginal contour of these particular indirect restorations. Thus, the aim of this study is to develop a novel design of the veneers deemed to augment the interfacial adhesive forces and, furthermore, to investigate the bonded interfaces by using optical coherence tomography.
\end{abstract}

Keywords: dental veneers, novel design, sinusoidal preparation, bonded interfaces, adhesive properties, Optical Coherence Tomography (OCT).

\section{INTRODUCTION}

Aesthetic dentistry has become one of the most popular fields of nowadays dentistry, as a beautiful smile represents one of the best trigger for a young and healthy appearance. Thus, dentists have to satisfy the patients' high expectations. Indirect dental veneers and the wide range of highly esthetic dental materials that are developed are a method to meet these expectations.

Since the bonding of the first ceramic dental veneer in 1930 by Dr. Charles Pincus, thousands of experimental studies have been conducted in order to enhance both mechanical and cosmetic properties. Despite the numerous studies, only a certain, classical appearance of these prosthetic treatment options has been considered in literature, with a linear contour, i.e., a straight and sheer contact with the adjacent dental structure [1]. Although this linear design has the advantage of simplicity, it has the major drawback of a smaller contact surface with the adjacent enamel, and also of the large amount of sound tooth removal [2,3]. As a consequence of the former, the retention itself is due mainly to the mechanical properties of the dental adhesives.

Chemical adhesion no longer represents a drawback in the long-term dental treatments, since considerable enhancements have been brought to the components and clinical properties of dental adhesives. Therefore, the adhesion of composite resins to the etched enamel exceeds nowadays $20 \mathrm{MPa}$, which is a good value for ensuring optimal retention and less microleakage of most restorative procedures [4]. However, one always wants to augment adhesive forces; this can be achieved using several methods: (i) by increasing the enamel surface aimed to interact with the resin; (ii) by exposing the organic part of the enamel; (iii) by clearing the superficial structures of the enamel; (iv) by adding polar phosphate groups on the enamel surface [5].

*maroiualexandra@gmail.com

Seventh International Conference on Lasers in Medicine, edited by Darinca Carmen Todea,

Adrian Gh. Podoleanu, Virgil-Florin Duma, Proc. of SPIE Vol. 10831, 1083100

(C) 2018 SPIE · CCC code: 1605-7422/18/\$18 · doi: 10.1117/12.2282648

Proc. of SPIE Vol. 10831 1083100-1 
The aim of this paper is to tackle with the debonding of the indirect dental veneers due to the classical linear marginal contour - issue (i) in the above discussion. In order to find a technical solution to this particular problem, we have developed a sinusoidal marginal design which is expected to display three major advantages: (a) higher retention due to the increased microretentions between the dental structure and the dental veneer, (b) higher adhesion due to larger superficial contact at the interface, and (c) a more accurate positioning of the veneers in situ during the luting procedure.

In addition to this, the sinusoidal bonded interfaces have been investigated in terms of external and internal adaptation of the novel veneers to the dental support by using Optical Coherence Tomography (OCT) working in Time Domain (TD). This particular sinusoidal dental veneer, subject of a recent patent request of our group [6], has not been described in the literature before, to the best of our knowledge.

\section{METHODOLOGY}

\subsection{Performing the sinusoidal tooth preparation}

A number of 20 human extracted teeth have had their vestibular face specifically prepared in order to consequently develop 20 indirect dental veneers by using different laboratory technologies. Thus, the novel design of the preparation consists of three sinusoidal marginal lines which outline the contour of the facial dental veneers, with the proximal limits positioned just before the interdental contact (Fig. 1).

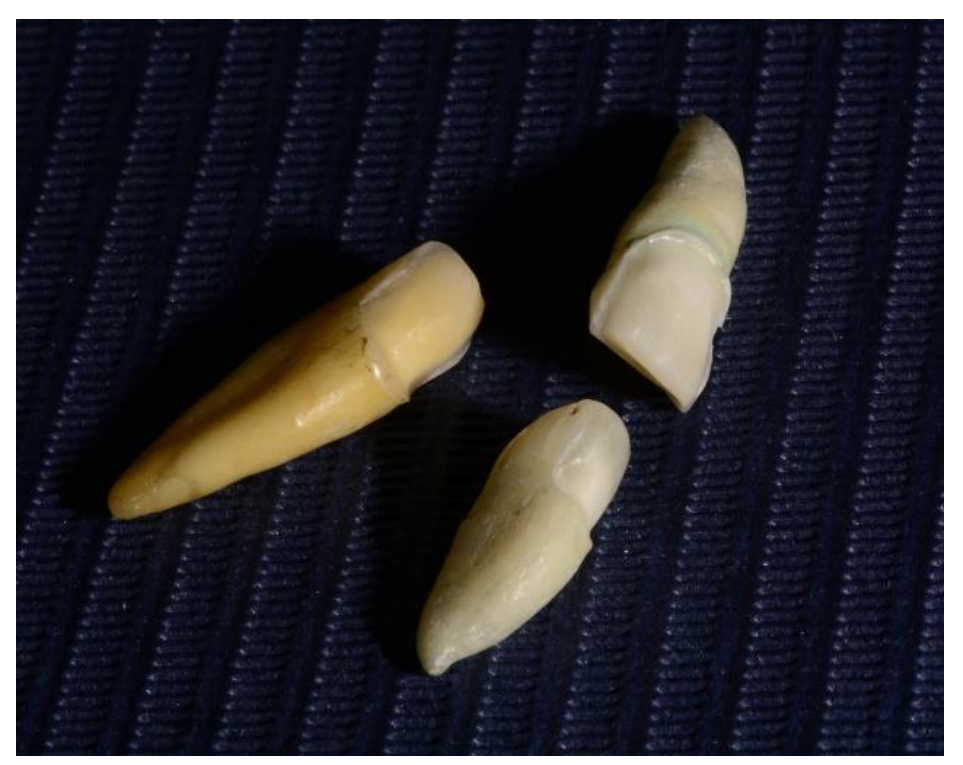

Figure 1. The facial and proximal aspect of the sinusoidal preparation that characterizes the crenellated veneers.

The height of the crenellated lines is correlated with the type of the tooth, namely: $2-2.5 \mathrm{~mm}$ for the lateral incisors and inferior central incisors, and 2.5-3 mm for the central upper incisors and for the canines. The depth of the sinusoidal proximal margins decreases progressively from $0.6-0.8 \mathrm{~mm}$ in the gingival third, to $0.4-0.6 \mathrm{~mm}$ in the middle third, and to 0.3-0.4 $\mathrm{mm}$ in the incisal third (Fig. 2).

The buccal face of the tooth is reduced by $0.5-0.8 \mathrm{~mm}$ and the incisal margin by $1 \mathrm{~mm}$ in order to provide an aesthetic final restoration. Thus, the crenellated dental veneers that we have propose in a patent [6] have a specific design that fits with the particular preparation of the tooth: three sinusoidal proximal margins with different heights and depths, 0.5-0.8 $\mathrm{mm}$ buccal thickness and $1 \mathrm{~mm}$ incisal thickness. Sinusoidal-bonded interfaces represent the major area for investigations in terms of external and internal adaptation of the crenelated veneer to the enamel and the Optical Coherence Tomography (OCT) tool [7-11] is used for evaluating the integrity and quality of these bonded joints. 


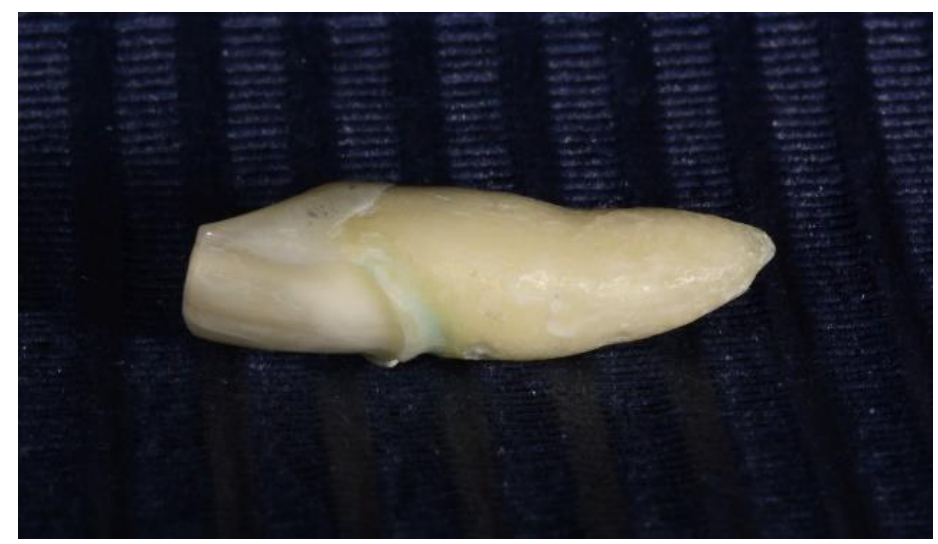

Figure 2. The particular proximal margin with its sinusoidal direction and variable depth.

\subsection{Elaborating the crenelated dental veneers}

In order to evaluate the precision of different laboratory procedures for elaborating the crenelated veneers and their internal marginal fit to the dental support, we divided the 20 final restorations in 3 experimental groups (Fig. 3), namely: Group 1, 7 lithium disilicate glass-ceramic veneers (IPS e.max Press); Group 2, 7 highly cross-linked polymeric veneers using CAD-CAM technique (Telio CAD); Group 3, 6 highly-cross-linked polymeric veneers using the cold technique (Telio Lab), all of them displaying a sinusoidal marginal contour (Fig. 4).

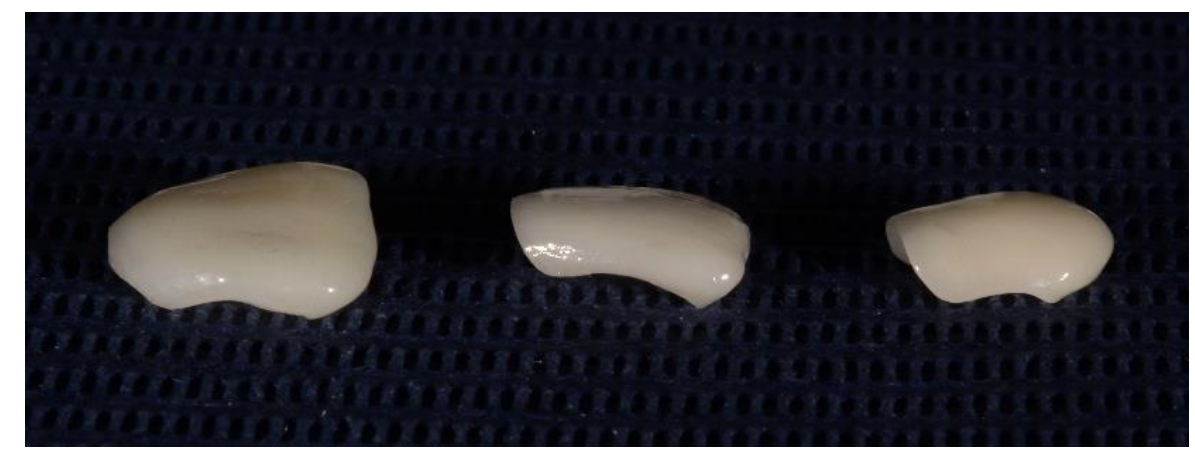

Figure 3. The proximal aspect of the final veneers (IPS e.max Press, Telio CAD, and Telio Lab) with their sinusoidal marginal contour.

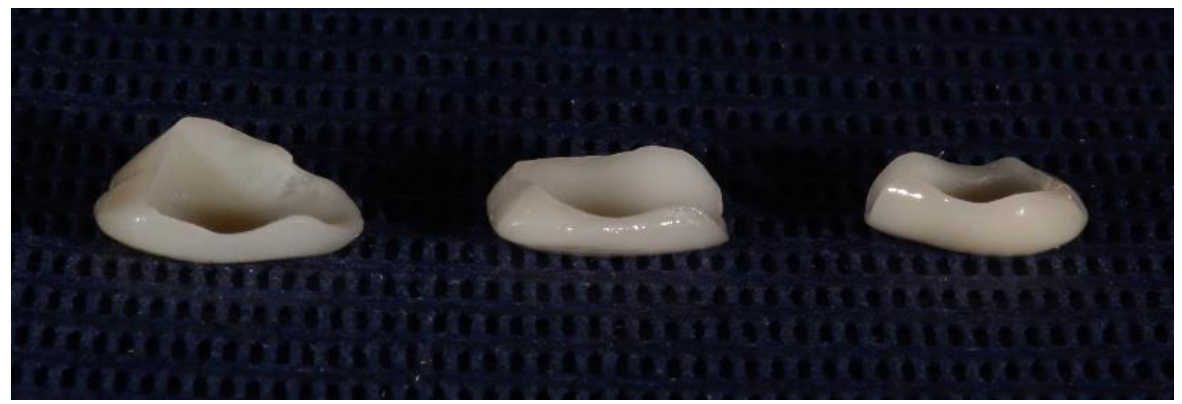

Figure 4. The proximal and internal aspect of the crenelated veneers elaborated by three different laboratory procedures. 


\subsection{Luting procedure}

The luting protocol consisted of proper conditioning of the veneers' internal surface, namely: sandblasting and etching with 5\% hydrofluoric acid for $20 \mathrm{~s}$ - for Group 1 and sandblasting for Group 2 and Group 3; applying the primer (Monobond Plus) for $60 \mathrm{~s}$ and the adhesive (Adhese Universal) onto all internal surfaces. The pre-treatment of the dental support included etching the enamel with phosphoric acid for $20 \mathrm{~s}$ and applying the adhesive (Adhese Universal). The luting cement (Variolink Esthetic LC) was afterwards dispensed on both enamel and restoration surfaces and polymerized for $40 \mathrm{~s}$ (iLED Woodpecker 2000mW/cm²) (Fig. 5).

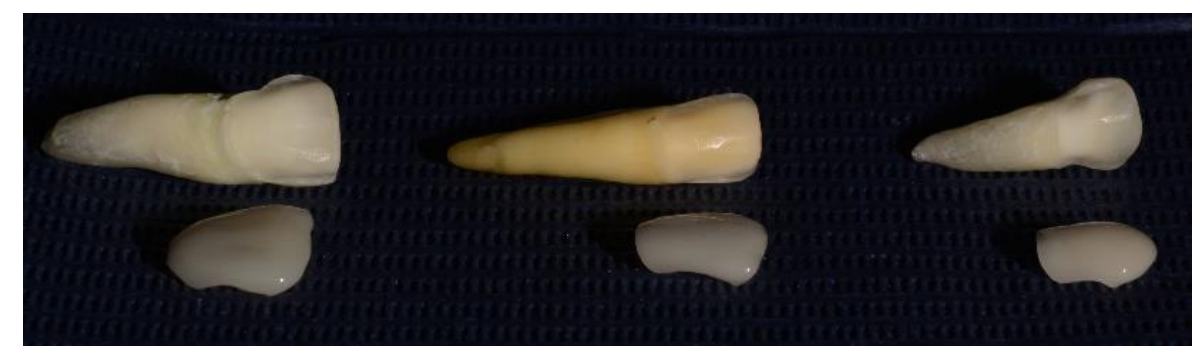

Figure 5. Three out of the twenty crenellated veneers, representing the three experimental groups prepared for the luting procedure.

\subsection{Analysis of the marginal sinusoidal bonded joints}

The investigation of the bonded joints (Fig. 6) was performed by using an in-house developed OCT system working in Time Domain (TD), with a center wavelength of $1500 \mathrm{~nm}$, performing B-scans/cross-section, in order to identify interfacial defects that may be caused by either inhomogeneous cement layer or improper fabrication of the veneers. These B-scans triggered 110 slices for every specimen, allowing us to adequately evaluate the integrity and quality of the bonded surfaces.

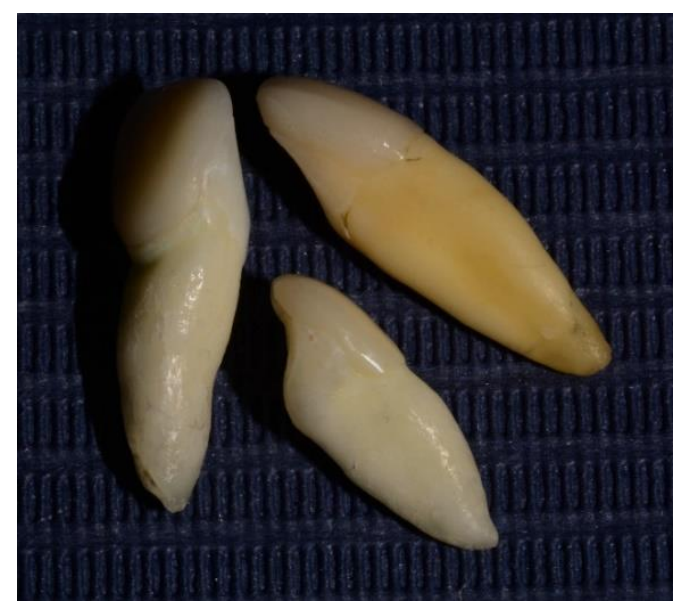

Figure 6 . The sinusoidal bonded interfaces displayed by the three experimental groups are investigated with OCT.

\section{RESULTS}

The bonded interfaces of all three experimental groups (IPS e.max Press, Telio CAD and Telio Lab) have been investigated using OCT and the 110 slices displayed accurately interfacial structures with no inner defects of the cement or veneers. The cement layer had the same homogenous thickness all over the sinusoidal junctions, thus proving a proper internal and marginal adaptation of the crenelated veneers to the enamel (Figs. 7, 8, and 9). 
According to the images achieved using OCT investigations, the internal and external adaptation of the crenelated veneers to the dental support have been fine, with no gaps or inner defects identified along the interfaces. Therefore, the failure rate due to marginal percolation, secondary decays, or sheer detachments of the veneers may be significantly reduced by elaborating this particular type of indirect restorations.

(a)

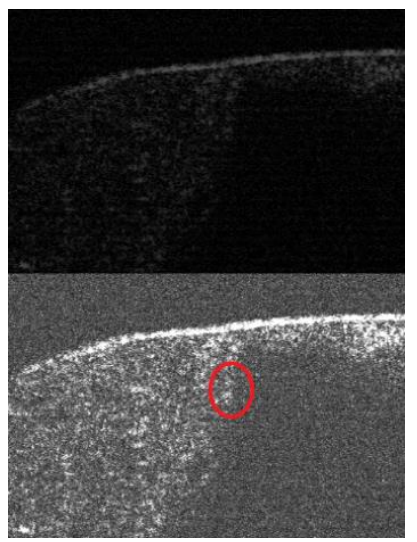

(c)

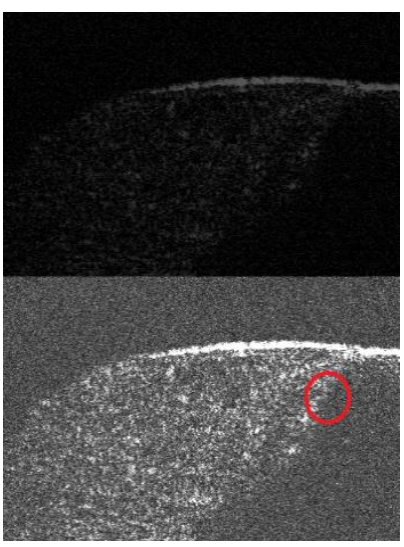

(b)

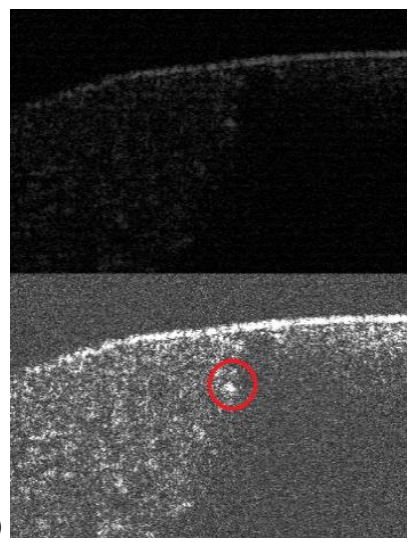

(d)

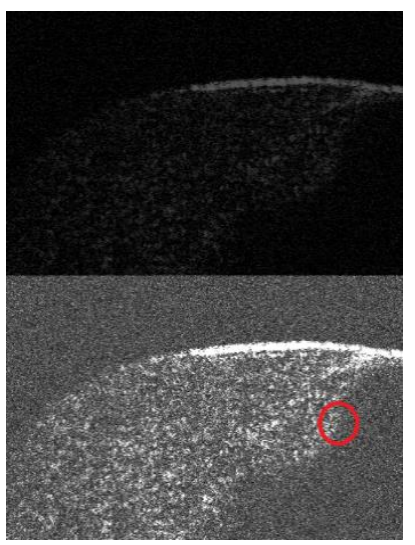

Figure 7. Telio CAD: OCT B-scans, lateral view of the bonded junction, no defects observed: (a) section 2/110; (b) section 37/110; (c) section 73/110; (d) section 104/110; the red circle represents the junction between the crenellated veneer (left) and the hard tissues (right).

(a)

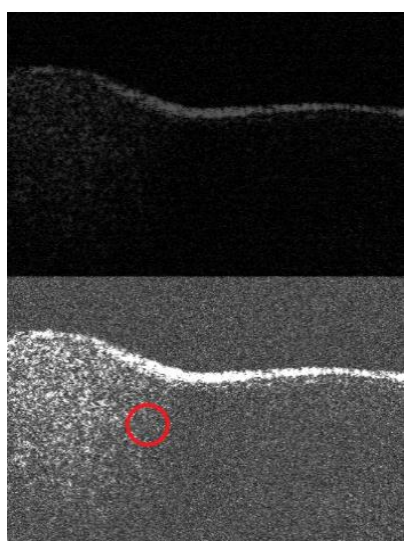

(b)

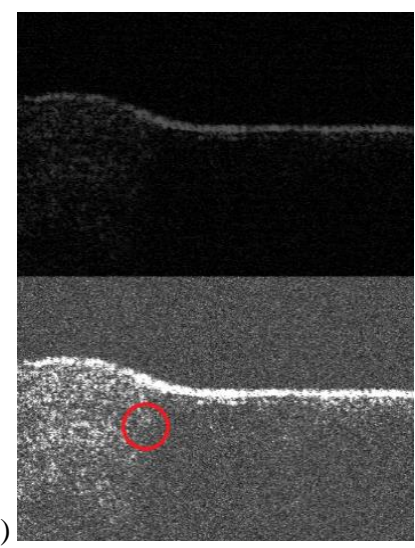


(c)

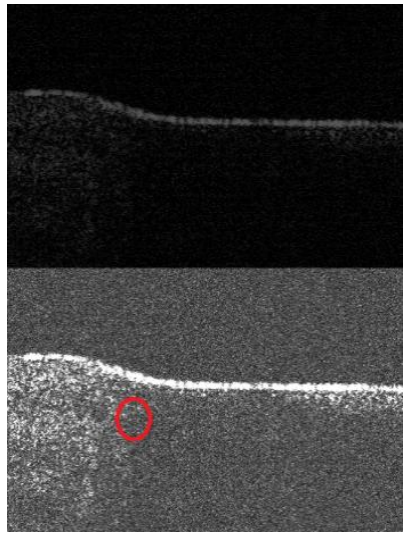

(d)

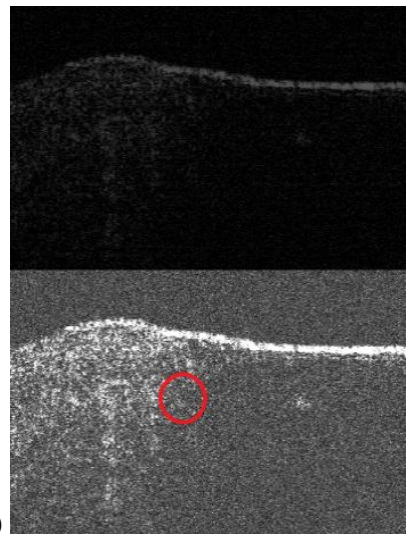

Figure 8. E.max Press: OCT B-Scans, lateral view of the bonded junction, no defects observed: (a) section 5/110; (b) section 28/110; (c) section 47/110; (d) section $103 / 110$; the red circle represents the junction between the crenellated veneer (left) and the hard tissues (right).

(a)

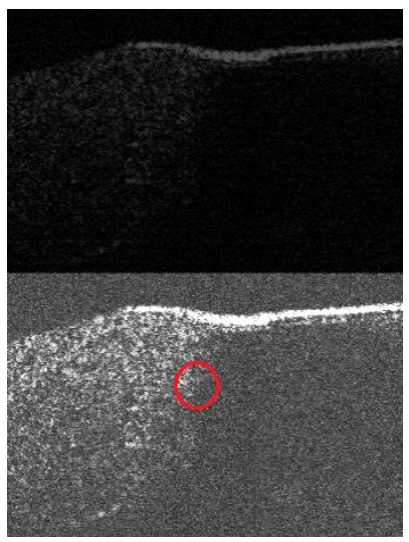

(c)

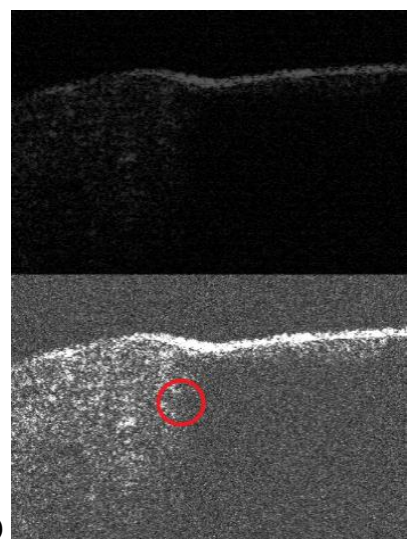

(b)

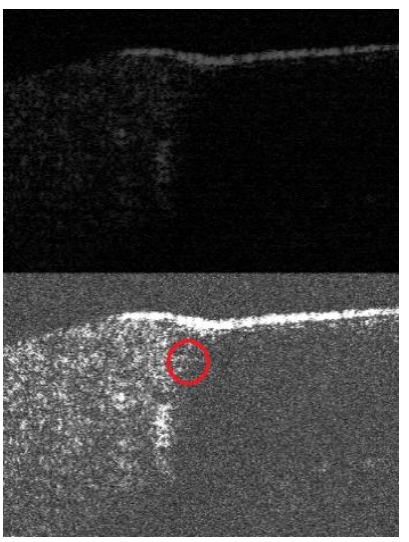

(d)

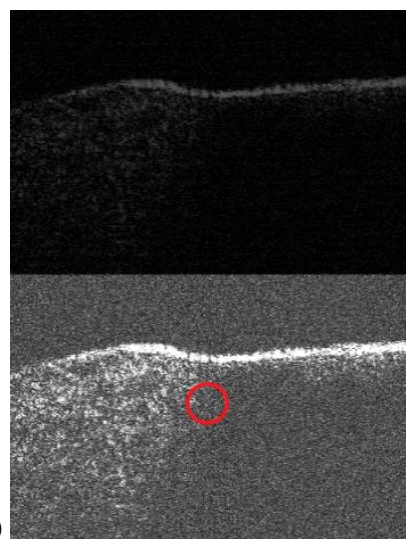

Figure 9. Telio Lab: OCT B-Scans, lateral view of the bonded junction, no defects observed: (a) section 7/110; (b) section 38/110; (c) section 76/110; (d) section 99/110; the red circle represents the junction between the crenellated veneer (left) and the hard tissues (right). 


\section{CONCLUSIONS}

The results of our experimental study demonstrated that OCT can successfully act as a valuable tool for interface monitoring. Moreover, it can be used for further investigation of the novel sinusoidal joints in terms of integrity and longevity, thus spreading its applicability in the prosthetics domain.

Apart from this, the sinusoidal veneer preparations that, to our knowledge, we have introduced (patent pending) provide accurate bonded joints, with no internal defects, thus showing potential for enhancing the adhesive and biomechanical properties of the final restorations. In addition to this, the crenelated veneers seem to represent a successful long-term treatment option in esthetic dentistry and they may represent the golden option for diminishing the detachment rate of the veneers from the dental support.

\section{ACKNOWLEDGMENTS}

This work was supported by the Romanian National Authority for Scientific Research, CNDI-UEFISCDI project PED PN-III-P2-2.1-PED-2016-1937 (http://3om-group-optomechatronics.ro/).

\section{REFERENCES}

[1] Gurel G., [The Science and Art of Porcelain Laminate Veneers], Quintessence Publishing Co. Ltd., 258-285 (2003).

[2] Castelnuovo J., Tjan, A., Philips K., Nicholls J., "Fracture load and mode of failure of ceramic veneers of different preparations", The Journal of Prosthetic Dentistry, 83, 171-180 (2010).

[3] Edelhoff D., Sorensen J., "Tooth structure removal associated with various preparations designs for anterior teeth", The Journal of Prosthetic Dentistry 87, 503-509 (2011).

[4] Romînu M., Florița Z., Ianeș C., Bratu D., [Materiale Dentare], Brumar, 272-278 (2003).

[5] Buonocore, M.G., "A simple method of increasing the adhesion of acrylic filling materials to enamel surfaces, J. Dent. Res., 34, 849-853 (1955).

[6] Măroiu A.-C., Sinescu C., Romînu M., Negrutiu M.-L., Rusu L.-C., Levai, C.-M., PATENT RO 131840 A0, A 2017 00028/30.05.2017, BOPI no. 5/2017.

[7] Huang, D., Swanson, E. A., Lin, C. P., Schuman, J. S., Stinson, W. G., Chang, W., Hee, M. R., Flotte, T., Gregory, K., Puliafito, C. A., and Fujimoto, J. G., “Optical coherence tomography," Science 254 (5035), 1178-1181 (1991).

[8] Wojtkowski, M. "High-speed optical coherence tomography: basics and applications," Applied Optics 49, D30-D61 (2010).

[9] Canjau, S., Todea, C., Negrutiu, M. L., Sinescu, C., Topala, F. I., Marcauteanu, C., Manescu, A., Duma, V.-F., Bradu, A., Podoleanu, A. Gh., "Optical Coherence Tomography for Non-Invasive ex vivo Investigations in Dental Medicine - a Joint Group Experience (Review),” Modern Technologies in Medicine 7, 97-115 (2015).

[10] Oancea, R., Bradu, A., Sinescu, C., Negru, R. M., Negrutiu, M. L., Antoniac, I., Duma, V.-F., and Podoleanu, A. Gh., Assessment of the sealant/tooth interface using optical coherence tomography, J. of Adhesion Science and Technology 29(1), 49-58 (2015).

[11] Sinescu, C., Negrutiu, M. L., Bradu, A., Duma, V.-F., and Podoleanu, A. Gh., "Noninvasive quantitative evaluation of the dentin layer during dental procedures using Optical Coherence Tomography," Computational and Mathematical Methods in Medicine, Paper ID 709076 (2015). 